\title{
Conducting High Impact Research with Limited Financial Resources (While Working From Home)
}

\author{
Paul H. P. Hanel \\ University of Essex, University of Bath
}

\begin{abstract}
The Covid-19 pandemic has far-reaching implications for researchers. For example, many researchers cannot access their labs anymore and are hit by budget-cuts from their institutions. Luckily, there are a range of ways how highquality research can be conducted without funding and face-to-face interactions. In the present paper, I discuss nine such possibilities, including meta-analyses, secondary data analyses, web-scraping, scientometrics, or sharing one's expert knowledge (e.g., writing tutorials). Most of these possibilities can be done from home, as they require only access to a computer, the internet, and time; but no state-of-the art equipment or funding to pay for participants. Thus, they are particularly relevant for researchers with limited financial resources beyond pandemics and quarantines.
\end{abstract}

Keywords: resources; meta-analysis; secondary data-analysis; Covid-19

Lower student numbers and a general economic recession caused by global quarantine measures to control the Covid-19 pandemic are putting a lot of pressure on universities and researchers (Adams, 2020). For example, lab access as well as research budgets are suspended, and recruitment of diverse samples, even online, might be more difficult (Lourenco \& Tasimi, 2020). The lack of funding can hamper the quantity and quality of research output and cause numerous issues. Indeed, early career researchers identified having few resources as a major reason they struggle with publishing and therefore advancing their careers (e.g., Lennon, 2019; Urbanska, 2019). Furthermore, a lack of resources and funding can have a detrimental effect on the mental health of PhD-students (Levecque et al., 2017) and academic staff (Gillespie et al., 2001). However, while having substantial resources arguably facilitates primary research (i.e., researchers collecting their own data), it is possible to conduct high-impact and high-quality re- search with little or no funding as well while working remotely from home.

In this paper, I provide nine examples of how highimpact research in biomedical and social sciences can be conducted with limited materialistic resources. That is research which is published in prestigious journals (e.g., journals that are among the top $25 \%$ in a given field according to Scopus). The list of examples presented is neither meant to be exhaustive nor representative. Nevertheless, I am hoping that the examples provided can inspire researchers to think of new research questions or methods and allow them to take some pressure off themselves. I discuss how people can conduct highimpact research using information provided within published work, with data collected by others (secondary data analysis), with researcher's expertise and interests (e.g., tutorials), as well as with simulation studies. Table 1 provides an overview of the nine approaches which are discussed in detail below. 
Table 1

How to conduct high impact research with limited resources: An overview

\begin{tabular}{|c|c|c|c|}
\hline Type of research & Summary & Example papers & Introductory texts \\
\hline Meta-analysis & $\begin{array}{l}\text { A quantitative review of the litera- } \\
\text { ture }\end{array}$ & $\begin{array}{l}\text { Cuijpers et al. (2013) Webb } \\
\text { et al. (2012) }\end{array}$ & $\begin{array}{l}\text { Borenstein et al. (2009); } \\
\text { Cheung and Vijayakumar } \\
\text { (2016); Moher et al. (2009) }\end{array}$ \\
\hline Scientometrics & Analysis of scientific publication & $\begin{array}{l}\text { Fanelli (2010a); Leimu and } \\
\text { Koricheva (2005) }\end{array}$ & $\begin{array}{l}\text { Leydesdorff and Milojević } \\
\text { (2013) }\end{array}$ \\
\hline $\begin{array}{l}\text { Network and Cluster } \\
\text { Analysis }\end{array}$ & $\begin{array}{l}\text { Analysing the relations of objects } \\
\text { (e.g., researchers, journals) with } \\
\text { each other }\end{array}$ & $\begin{array}{l}\text { Cipresso et al. (2018); Wang } \\
\text { and Bowers (2016) }\end{array}$ & Costantini et al. (2015) \\
\hline $\begin{array}{l}\text { Data collected by or- } \\
\text { ganisations }\end{array}$ & $\begin{array}{l}\text { Typically large datasets that are } \\
\text { openly accessible in the internet }\end{array}$ & $\begin{array}{l}\text { Hanel and Vione (2016); } \\
\text { Ondish and Stern (2017) }\end{array}$ & $\begin{array}{l}\text { Cheng and Phillips (2014); } \\
\text { Rosinger and Ice (2019) }\end{array}$ \\
\hline Re-using data & $\begin{array}{l}\text { Using data collected by researchers; } \\
\text { typically main findings are already } \\
\text { published. }\end{array}$ & Coelho et al. (2020) & \\
\hline Web-scraping & $\begin{array}{l}\text { Extracting or harvesting data from } \\
\text { the internet (e.g., social media) }\end{array}$ & $\begin{array}{l}\text { Guess et al. (2019); Preis et } \\
\text { al. (2013) }\end{array}$ & $\begin{array}{l}\text { Michel et al. (2011); Paxton } \\
\text { and Griffiths (2017) }\end{array}$ \\
\hline Tutorials & Sharing one's expert knowledge & $\begin{array}{l}\text { Clifton and Webster (2017); } \\
\text { Weissgerber et al. (2015) }\end{array}$ & \\
\hline Theoretical papers & Developing new theories & $\begin{array}{l}\text { Ajzen (1991); } \quad \text { Festinger } \\
\text { (1957) }\end{array}$ & $\begin{array}{l}\text { Van Lange (2013); Smaldino } \\
\text { (2020) }\end{array}$ \\
\hline Simulation Studies & $\begin{array}{l}\text { Computer experiments, creating } \\
\text { data }\end{array}$ & $\begin{array}{l}\text { May and Hittner (1997); } \\
\text { Schmidt-Catran and Fair- } \\
\text { brother (2016) }\end{array}$ & $\begin{array}{l}\text { Beaujean (2018); Feinberg } \\
\text { and Rubright (2016); Morris } \\
\text { et al. (2019) }\end{array}$ \\
\hline
\end{tabular}

Of course, whether it will be easy or difficult to acquire the necessary skills to write a paper within any of the nine approaches discussed below depends on a range of factors such as previous experience, complexity of the research question, and data availability to answer a specific research question. That is, it can be easier to publish a paper using for example secondary data because no data collection is required, but if a researcher is unfamiliar with specific statistical analyses such as multi-level modeling and the relevant literature, it might take longer than collecting primary data and writing a paper up.

\section{Information Provided Within Articles}

\section{Meta-analyses}

A meta-analysis is a quantitative review of the literature on a specific topic. The main aims are to estimate the strength of an effect across studies, test for moderators, publication bias, and to identify gaps in the literature (Borenstein et al., 2009; Simonsohn et al., 2015). For example, researchers might be interested in testing which emotion regulation strategy works best (Webb et al., 2012) or whether psychotherapy is better than pharmacotherapy in treating depressive and anxiety disor- ders (Cuijpers et al., 2013).

To perform a meta-analysis, researchers tend to start with a systematic literature review ${ }^{1}$, identify relevant articles and ideally unpublished studies, extract descriptive statistics (e.g., sample size, descriptive statistics) and information of relevant moderators (e.g., country of origin, sample type), and finally meta-analyse across samples (Cheung \& Vijayakumar, 2016). Thus, researchers need only a computer and access to the internet to perform a meta-analysis ${ }^{2}$. Nevertheless, a meta-analysis is hard work and a range of pitfalls, such

\footnotetext{
${ }^{1}$ A systematic review alone without a quantitative synthesis can be useful as well. For example, when there are only a few or too diverse papers published in a specific topical area, a qualitative summary only can be informative.

${ }^{2}$ Many research projects in general and meta-analyses in particular can benefit from collaborations. For example, any coding of studies is ideally done by at least two researchers. Finding reliable collaborators can be an issue for people with a smaller research network, especially in times when labs are closed, conferences cancelled, and home office is encouraged. There are many ways how potential collaborators can be identified (Sparks 2019). One is to first identify researchers who already published relevant articles or graduate students that are listed on the lab pages of more senior researchers and start follow them on social media to get an impression of their
} 
as an unsystematic literature review, must be avoided. Luckily, guidelines exist which help to overcome pitfalls (e.g., PRISMA guidelines) (Moher et al., 2009) as well as how to reduce publication bias (Stanley \& Doucouliagos, 2014), and powerful software can facilitate the statistical analysis and visualisations (e.g., the R-package metafor) (Viechtbauer, 2010). Also, pre-registration of meta-analyses is possible (Quintana, 2015; Stewart et al., 2012).

Meta-analyses are useful for many disciplines because they provide a robust effect size estimate of a specific research question. Also, meta-analyses typically attract more citations than empirical studies (Patsopoulos et al., 2005). Meta-analyses that identify moderators or develop new taxonomies based on the literature can be especially influential (Webb et al., 2012). If meta-analyses already exist in a given subfield, researchers can consider performing a second-order metaanalysis: A meta-analysis across meta-analyses to get even more robust effect size estimates (Hyde, 2005) or to test for moderators such as cultural factors (Fischer et al., 2019). Additionally, meta-analyses come with secondary benefits for meta-analysts themselves. Everyone who has performed a meta-analysis knows that identifying the relevant information such as descriptive statistics or effect sizes in empirical articles can easily get frustrating because authors often do not report sufficient information. This can mean that otherwise perfectly suitable studies cannot be included in a metaanalysis. Thus, every PhD-student in biomedical and social sciences working on a quantitative research question, might want to consider performing a meta-analysis at the beginning of their program to teach them the importance of reporting detailed results and ideally also of sharing the (anonymised) data openly.

One objection against the claim that every researcher with a computer and internet access can perform a meta-analysis, might be that particularly less affluent institutions can not pay the high subscription fees for many scientific journals. However, as the number of pre-prints and open access journals are increasing, paywalls become less of an issue. Further, researchers from less affluent institutions can collaborate with colleagues from institutions with access to the required journals. Finally, while legally questionable, researchers have found a way to bypass the paywall of most scientific publishers (Bohannon, 2016).

\section{Scientometrics}

Scientometrics is an interdisciplinary scientific field that analyses scientific publication trends using various statistical methods. There are countless ways that publications can be analysed. I will discuss a few of them in this and the next section. For example, one line of publications investigates how often so-called statistically significant findings occur: Are 'positive' results increasing "down the hierarchy of the sciences" (Fanelli, 2010a), does publication pressure increases scientists' bias (Fanelli, 2010b), or are p-values just below .05 occurring more frequently than one would expect assuming no publication bias (Simonsohn et al., 2015)?

A prominent example of scientometrics is citation analysis. For example, what predicts whether a scientific article gets cited? Is it whether it is published open access (McKiernan et al., 2016) or whether sample sizes are large (Hanel \& Haase, 2017)? All relevant information to address these questions can be extracted from the articles of a specific scientific (sub-)field and sometimes even from meta-analyses (Hanel \& Haase, 2017). Typically, questions such as these are investigated separately in each subfield such as internal medicine (Van der Veer et al., 2015).

Similar research questions can be tested with citations aggregated on a journal level. The amount of citations articles published in the last 2, 3, or 5 years in a specific journal are averaged and used as quality indicator of that journal (i.e., the so-called Journal Impact Factor or, more recently, Cite Score) (Teixeira da Silva \& Memon, 2017). However, it is an empirical question in its own right whether these quality indicators are associated with other quality indicators of empirical studies (Brembs, 2018), and whether there are unintended consequences of ranking journals based on alleged quality (Brembs et al., 2013). Research questions such as these, and others like them, can again be tested with limited resources as they often only require the coding of published articles (e.g., on some quality indicators).

Furthermore, some journals are asking reviewers to assess the quality of a manuscript quantitatively when providing their review. If one has access to how reviewers evaluate manuscripts it is possible to assess whether reviewers agree on the quality of the manuscript (Bornmann et al., 2010), or whether reviewers (Reinhart, 2009) can predict how well a paper or researchers get cited in the following years.

\section{Network and Cluster Analyses of the Published Lit- erature}

Yet another way to perform research at low costs is to perform network and cluster analyses. A network "is an

views and beliefs on various issues. Then reach out to them via email to gauge their general interest and, in case of a positive reply, schedule a video chat. If this is going well, it might be useful to discuss early on who contributes what and authorships. Who does what? Who gets to be first author? It is worth keeping in mind that shared (first) authorships are possible. 
abstract representation of a system of entities or variables (i.e., nodes) that have some form of connection with each other (i.e., edges)" (Dalege et al., 2017, p. 528). Nodes can represent a variety of things including people, journals, or keywords. In short, network analyses typically reveal how strongly objects are associated. For example, from combing through keywords, journal names, citation counts, or country of origin of authors from hundreds or thousands of articles, it is possible to identify emerging themes and track a disciplines evolution. This can show which keywords are more frequently used together, which journals cite each other (journal citation network analysis), or researchers from which countries collaborate together more frequently (Cipresso et al., 2018). In addition to this, these analyses also allow researchers to identify potential gaps in the literature (e.g., if two or more keywords are not linked in a keyword network analysis, this might indicate a potential gap in the literature). Finally, moving beyond network analysis, extracting the full text of scientific articles can be used to analyse their readability (Plavén-Sigray et al., 2017) or to estimate the accuracy of the reported statistical information (Nuijten et al., 2015), for instance.

\section{Secondary Data Analysis}

\section{Data Made Available by (Research) Organisations}

Over the past decades, the number of large, openly available surveys relevant to the social sciences and researchers interested in the mental health of people has grown rapidly. Several of them are conducted in national representative samples in just one country (e.g., British Election Study, American National Election Studies), while others contain data from up to 70 countries (e.g., European Social Survey, World Values Survey). There is also a range of open datasets that might be of interest to biomedical researchers and neuroscientists such as the Human Connectome Project which includes anatomical and diffusion neuroimaging data; the Star*D project which includes antidepressant treatment of patient diagnosed with major depressive disorder, or the UK biobank which contains health information of 500,000 volunteer participants.

Many of these surveys are conducted every few years. Since the surveys are openly and freely available to researchers and contain many variables relevant to social scientists, they can be used to answer a range of research questions. Research questions addressed by past research include: whether student samples provide a good estimate of the general public (Hanel \& Vione, 2016) or whether social trust and self-rated health are positively correlated (Jen et al., 2010), and whether scales are invariant across groups of people (Cieciuch et al., 2017).

Additionally, it's possible to combine data from large surveys with other data. For example, Nosek et al. (2009) correlated implicit gender-science stereotypes from the Project Implicit with the gender differences in science and math achievements from the Trends in International Mathematics and Science Study (Gonzales et al., 2003). Basabe and Valencia (2007) correlated the country averages of Hofstede's (2001) cultural dimensions, Inglehart's (Inglehart \& Baker, 2000) values as measured by the World Values Survey, and Schwartz's (2006) cultural value dimensions, with indices of human development provided in the United Nations Report (e.g., 2014) and De Riviera's (2004) culture of peace dimensions. Such analyses allow to identify, for example, what predicts whether a country is more likely to engage in wars and supress its own population. As all the prior mentioned datasets are openly available, it is relatively easy to reproduce all analyses and come up with new research questions that can be answered with these datasets. Further, it is possible to pre-register secondary data analysis (Van den Akker et al., 2019).

The complexity of statistical analysis depends on the research question and data. For example, testing hypothesis with large $(\mathrm{N}>40,000)$ datasets containing data from various countries typically requires multilevel modeling, because participants are nested within countries (for an example paper see Rudnev \& Vauclair, 2018). In contrast, when two or more datasets have been combined, and, for example, only country-level data is available, researchers typically rely on correlation and regression analyses (e.g., Basabe \& Valencia, 2007; Inman et al., 2017). Recommendations for performing secondary data analyses exist, for example, for social studies (Fitchett \& Heafner, 2017), medical sciences (Cheng \& Phillips, 2014), human biology (Rosinger \& Ice, 2019), and qualitative research (Sherif, 2018).

\section{Reusing Data}

This point is similar to the one above, except that it focuses solely on reusing data collected by either researchers' own lab-group or that were shared by other researchers. Typically, the data were collected to answer some pre-defined research question, but not for the additional analyses someone thought about only after data collection. Further, if one has access to several similar datasets that also included some demographic information which may have been reported but were not the focus of the main paper(s), the datasets can be combined and reanalysed to test for differences and similarities between the demographic groups on several of the 
primary variables (assuming this analysis has not been reported in the primary papers). In a similar refrain, if several primary studies included a scale with, as a rule-of-thumb, more than eight-items per dimension, it is worth considering to test whether only some of the items of each dimension is as reliable and valid as the original scale (Coelho et al., 2020).

Both types of research questions (comparisons across demographic groups and scale validation) along with several other ones, can also entirely be addressed with datasets openly shared by researchers. Google has created a search engine that searches for open datasets (https://datasetsearch.research.google.com/; see also https://dataverse.harvard.edu/) which can be directly used or combined with other datasets. To the best of my knowledge, the number of articles based on re-using data collected by other researchers is still very limited. However, since more and more researchers are sharing their data and search engines allow to identify potentially relevant datasets, the number of papers based on other researchers' data is likely increasing.

An additional way to reuse data is to verify the results of an already published article with the data collected by the original authors. The necessity of this is illustrated by an attempt to replicate 59 macroeconomic papers using the original data (Chang \& Li, 2017). Only 29 papers were replicated, even with the help of the original authors. Such an initiative would be very useful in other scientific fields too. But also replicating the results from single papers has been encouraged. For example, the journal Cortex has recently announced a new article type "Verification Reports" which reports independent replication of the research findings of a published article through repeating the original analyses. This is to "provide scientists with professional credit for evaluating one of the most fundamental forms of credibility: whether the claims in previous studies are justified by their own data" (Chambers, 2020, p. A1).

\section{Web-Scraping}

When people use social media or use a search engine, they produce data. Some of the traces people leave online can be relatively easy scrapped (i.e., extracted or harvested) and allow us to answer research questions we would not be able to answer with traditional approaches (cf. Paxton \& Griffiths, 2017). Webpages from which data can relatively easily obtained include: Twitter, Reddit, as well as Google Ngram Viewer, and Google Trends. For example, researchers used Twitter to test whether survey responses of social media use are accurate (Guess et al., 2019), and predictors of solidarity expressions with refugees (Smith et al., 2018). Further, Google Trends - which analyses how often people searched using Google for specific terms in one or all countries on a specific date - was used to test whether online health-seeking behaviour predicts influenza-like symptoms (Ginsberg et al., 2009) and whether Google searches predict stock market moves (Preis et al., 2013).

\section{Other Outputs}

\section{Tutorials}

To conduct high quality primary research, researchers often need to acquire specific skills. Examples of expert knowledge and skills that researchers may have, include recruiting participants from hard to reach populations, setting up testing equipment which often includes programming skills (e.g., in cognitive psychology or neuroscience), and analysing the data. Without a good mentor, helpful peers, or informative tutorials, acquiring such skills can be cumbersome. Sharing this knowledge by writing blogposts or peer-reviewed articles (e.g., tutorials) can therefore be very useful once we acquired some specialist expert knowledge. For example, what recruitment methods work well to get couples to participate in unpaid online or lab-studies (e.g., flyer distribution on places where people are waiting anyway such as train stations, schools, on campus, or targeting specific groups on social media)? What are best practices for writing reproducible code? How should data of a specific format be analysed? Writing a step-by-step tutorial (assuming this does not yet exist), ideally with some concrete examples, may be often cited and help to establish a reputation as an expert.

Previous tutorials focused on various statistical methods such as response surface analysis (Barranti et al., 2017), network analysis (Dalege et al., 2017), multilevel meta-analyses (Assink \& Wibbelink, 2016), or Bayesian statistics (Weaver \& Hamada, 2016); recommendations for data visualisation (Weissgerber et al., 2016), or web-scrapping (Bradley \& James, 2019); suggestions for open science practices (Allen \& Mehler, 2019); or how to use databases (Waagmeester et al., 2020). A more advanced type of tutorials concerns software packages, because they usually include computer code that assist others directly in performing a specific analysis, additional to a (peer-reviewed) article (Viechtbauer, 2010). Expert knowledge also allows researchers more easily to write commentaries on various topics. Popular commentaries include topics such as the scientific publication system (Lawrence, 2003) or cargo cult science (Feynman, 1974).

\section{Theoretical Papers}

Related to tutorials, scientists can integrate and advance research in theoretical papers. Theories are im- 
portant because they help us to see "the coherent structures in seemingly chaotic phenomena and make inroads into previously uncharted domains, thus affording progress in the way we understand the world around us" (Van Lange, 2013, p. 40). In contrast to tutorials which typically focus on solving specific problems such as conducting a specific analysis, theoretical papers can both solve problems through integrating apparently contradictory findings into one broader framework, but can also 'cause' problems through making novel predictions and is therefore crucial for new empirical discoveries (Higgins, 2004).

Prominent examples include the theory of planned behavior (Ajzen, 1985, 1991) which aims to explain planned human behaviour or cognitive dissonance theory (Festinger, 1957) which aims to explain how people deal with internal inconsistencies. However, developing a formalised and testable theory can be challenging. For example, van Lange (2013) argues that good theories should contain "truth, abstraction, progress, and applicability as standards" (p. 40) and provides recommendations how this can be done. Smaldino (2020) discusses various options how verbal theories can be translated into formal models.

\section{Simulation Studies}

Another way to get data without needing to conduct a study, is to simulate data from hundreds or often even thousands of studies using specialised statistical software such as the freely available program $\mathrm{R}$ (Feinberg \& Rubright, 2016). In a simulation study, data are generated that may or may not reflect real data. Thanks to the advances in processing capacities, many simulation studies can be done nowadays without needing to access a supercomputer. Simulation studies have been used to answer a range of questions, such as which mediation test best balances type-I error and statistical power (MacKinnon et al., 2002) and the pitfalls in specifying fixed and random effects in multilevel models (Schmidt-Catran \& Fairbrother, 2016).

The first step in a simulation study is typically to define the problem. For example, a researcher might be interested in exploring which, out of multiple tests that serve the same purpose, has the lowest type-I and typeII error rates. Other steps include making assumptions, simulating the data, evaluating the output, and finally disseminating the findings (for tutorials see Beaujean, 2018; Feinberg \& Rubright, 2016). In short, simulation studies are an effective way to conduct cheap research, but require advanced programming skills.

\section{Conclusion}

In the present paper, I provide suggestions of how impactful research can be conducted with limited resources and while working remotely. The above list is not meant to be exhaustive but will hopefully provide some examples that might inspire researchers to consider alternative ways to research phenomena they find interesting. Importantly, encouraging researchers to conduct research using more secondary data analysis does not disregard primary empirical research. However, it is sometimes not feasible for everyone to conduct well-powered empirical studies because of a limited amount of resources. Thus, being aware of alternative ways to conduct research can help researchers in this situation, to get to a point in which they can compete with researchers who have access to more resources (cf. Lepori et al., 2019). Ultimately, it might make science more egalitarian, because it also allows researchers from financially less well-situated institutions to publish in prestigious journals.

\section{Author Contact}

Paul Hanel, Department of Psychology, University of Essex, Colchester, United Kingdom. p.hanel@essex.ac.uk

Department of Psychology, University of Essex, Colchester, United Kingdom

Acknowledgements. I wish to thank Martha Fitch Little and Wijnand van Tilburg for useful comments on an earlier version of this paper.

\section{Conflict of Interest and Funding}

The author has no conflict of interest to declare. There was no specific funding for this project.

\section{Author Contributions}

This is a single author contribution.

\section{Open Science Practices}

This theoretical article contains no data, materials or analysis. The entire editorial process, including the open reviews, are published in the online supplement.

\section{References}

Adams, R. (2020, April 22). Coronavirus UK: Universities face $£ 2.5 \mathrm{bn}$ tuition fee loss next year. The Guardian. https://www.theguardian.com/education/2020 /apr/23/coronavirus-uk-universities-face-25bntuition-fee-loss-next-year 
Ajzen, I. (1985). From Intentions to Actions: A Theory of Planned Behavior. In J. Kuhl \& J. Beckmann (Eds.), Action Control: From Cognition to Behavior (pp. 11-39). Springer. https://doi.org/10.1007/978-3-642-69746-3_2

Ajzen, I. (1991). The theory of planned behavior. Organizational Behavior and Human Decision Processes, 50(2), 179-211. https://doi.org/10.1016/07495978(91)90020-T

Allen, C., \& Mehler, D. M. A. (2019). Open science challenges, benefits and tips in early career and beyond. PLOS Biology, 17(5), e3000246. https://doi.org/10.1371/journal.pbio.3000246

Assink, M., \& Wibbelink, C. J. M. (2016). Fitting three-level meta-analytic models in R: A step-by-step tutorial. The Quantitative Methods for Psychology, 12, 154-174. https://doi.org/10.20982/tqmp.12.3.p154

Barranti, M., Carlson, E. N., \& Côté, S. (2017). How to test questions about similarity in personality and social psychology research Description and empirical demonstration of response surface analysis. Social Psychological and Personality Science, 8(4), 465-475. https://doi.org/10.1177/1948550617698204

Basabe, N., \& Valencia, J. (2007). Culture of peace: Sociostructural dimensions, cultural values, and emotional climate. Journal of Social Issues, 63(2), 405-419. https://doi.org/10.1111/j.15404560.2007.00516.x

Beaujean, A. A. (2018). Simulating data for clinical research: A tutorial. Journal of Psychoeducational Assessment, 36(1), 7-20. https://doi.org/10.1177/0734282917690302

Bohannon, J. (2016). Who's downloading pirated papers? Everyone. Science, 352(6285), 508-512. https://doi.org/10.1126/science.352.6285.508

Borenstein, M., Hedges, L. V., Higgins, J. P. T., \& Rothstein, H. (2009). Introduction to meta-analysis. John Wiley \& Sons.

Bornmann, L., Mutz, R., \& Daniel, H.-D. (2010). A reliability-generalization study of journal peer reviews: A multilevel metaanalysis of inter-rater reliability and its determinants. PLoS ONE, 5(12), e14331. https://doi.org/10.1371/journal.pone.0014331

Bradley, A., \& James, R. J. E. (2019). Web Scraping Using R. Advances in Methods and Practices in Psychological Science. https://doi.org/10.1177/2515245919859535

Brembs, B. (2018). Prestigious Science Journals Struggle to Reach Even Average Reliability. Frontiers in Human Neuroscience, 12. https://doi.org/10.3389/fnhum.2018.00037

Brembs, B., Button, K., \& Munafò, M. (2013). Deep impact: Unintended consequences of journal rank. Frontiers in Human Neuroscience, 7, 291. https://doi.org/10.3389/fnhum.2013.00291

Chambers, C. D. (2020). Verification Reports: A new article type at Cortex. Cortex, 129, A1-A3. https://doi.org/10.1016/j.cortex.2020.04.020

Chang, A. C., \& Li, P. (2017). A Preanalysis Plan to Replicate Sixty Economics Research Papers That Worked Half of the Time. American Economic Review, 107(5), 60-64. https://doi.org/10.1257/aer.p20171034

Cheng, H. G., \& Phillips, M. R. (2014). Secondary analysis of existing data: Opportunities and implementation. Shanghai Archives of Psychiatry, 26(6), 371-375. https://doi.org/10.11919/j.issn.10020829.214171

Cheung, M. W.-L., \& Vijayakumar, R. (2016). A guide to conducting a meta-analysis. Neuropsychology Review, 26(2), 121-128. https://doi.org/10.1007/s11065-016-9319-z

Cieciuch, J., Davidov, E., Algesheimer, R., \& Schmidt, P. (2017). Testing for approximate measurement invariance of human values in the European Social Survey. Sociological Methods \& Research, 47(4), 665-686. https://doi.org/10.1177/0049124117701478

Cipresso, P., Giglioli, I. A. C., Raya, M. A., \& Riva, G. (2018). The Past, Present, and Future of Virtual and Augmented Reality Research: A Network and Cluster Analysis of the Literature. Frontiers in Psychology, 9. https://doi.org/10.3389/fpsyg.2018.02086

Clifton, A., \& Webster, G. D. (2017). An introduction to social network analysis for personality and social psychologists. Social Psychological and Personality Science, 8(4), 442-453. https://doi.org/10.1177/1948550617709114

Coelho, G. L. de H., Hanel, P. H. P., \& Wolf, L. J. (2018). The very efficient assessment of need for cognition: Developing a 6-Item version. Assessment. https://doi.org/10.1177/1073191118793208

Costantini, G., Epskamp, S., Borsboom, D., Perugini, M., Mõttus, R., Waldorp, L. J., \& Cramer, A. O. J. (2015). State of the aRt personality research: A tutorial on network analysis of personality data in R. Journal of Research in Personality, 54, 13-29. https://doi.org/10.1016/j.jrp.2014.07.003

Cuijpers, P., Sijbrandij, M., Koole, S. L., Andersson, G., Beekman, A. T., \& Reynolds, C. F. (2013). The efficacy of psychotherapy and pharmacotherapy in treating depressive and anxi- 
ety disorders: A meta-analysis of direct comparisons. World Psychiatry, 12(2), 137-148. https://doi.org/10.1002/wps.20038

Dalege, J., Borsboom, D., van Harreveld, F., \& van der Maas, H. L. J. (2017). Network analysis on attitudes: A brief tutorial. Social Psychological and Personality Science, 8(5), 528-537. https://doi.org/10.1177/1948550617709827

De Rivera, J. (2004). Assessing the Basis for a Culture of Peace in Contemporary Societies. Journal of Peace Research, 41(5), 531-548. https://doi.org/10.1177/0022343304045974

Fanelli, D. (2010a). "Positive" results increase down the hierarchy of the sciences. PloS One, 5(4), e10068. https://doi.org/10.1371/journal.pone.0010068

Fanelli, D. (2010b). Do pressures to publish increase scientists' bias? An empirical support from US States data. PLOS ONE, 5(4), e10271. https://doi.org/10.1371/journal.pone.0010271

Feinberg, R. A., \& Rubright, J. D. (2016). Conducting Simulation Studies in Psychometrics. Educational Measurement: Issues and Practice, 35(2), 36-49. https://doi.org/10.1111/emip.12111

Festinger, L. (1957). A theory of cognitive dissonance. Stanford University Press.

Feynman, R. P. (1974). Cargo cult science. Engineering and Science, 37, 10-13.

Fischer, R., Karl, J. A., \& Fischer, M. V. (2019). Norms Across Cultures: A Cross-Cultural Meta-Analysis of Norms Effects in the Theory of Planned Behavior. Journal of CrossCultural Psychology, 50(10), 1112-1126. https://doi.org/10.1177/0022022119846409

Fitchett, P. G., \& Heafner, T. L. (2017). Quantitative Research and Large-Scale Secondary Analysis in Social Studies. In Handbook of Social Studies Research (pp. 68-94). John Wiley \& Sons, Ltd. https://doi.org/10.1002/9781118768747.ch4

Gillespie, N. A., Walsh, M., Winefield, A. H., Dua, J., \& Stough, C. (2001). Occupational stress in universities: Staff perceptions of the causes, consequences and moderators of stress. Work \& Stress, 15(1), 53-72. https://doi.org/10.1080/02678370117944

Ginsberg, J., Mohebbi, M. H., Patel, R. S., Brammer, L., Smolinski, M. S., \& Brilliant, L. (2009). Detecting influenza epidemics using search engine query data. Nature, 457(7232), 1012-1014. https://doi.org/10.1038/nature07634

Gonzales, P. (2003). Highlights from the trends in international mathematics and science study (TIMSS) 2003.

Guess, A., Munger, K., Nagler, J., \& Tucker,
J. (2019). How accurate are survey responses on social media and politics? Political Communication, 36(2), 241-258. https://doi.org/10.1080/10584609.2018.150 4840

Hanel, P. H. P., \& Haase, J. (2017). Predictors of citation rate in psychology: Inconclusive influence of effect and sample size. Frontiers in Psychology, 8. https://doi.org/10.3389/fpsyg.2017.01160

Hanel, P. H. P., \& Vione, K. C. (2016). Do student samples provide an accurate estimate of the general public? PLOS ONE, 11(12), e0168354. https://doi.org/10.1371/journal.pone.0168354

Higgins, E. T. (2004). Making a theory useful: Lessons handed down. Personality and Social Psychology Review, 8(2), 138-145. https://doi.org/10.1207/s15327957pspr0802_7

Hofstede, G. (2001). Culture's consequences: Comparing values, behaviors, institutions and organizations across nations (2nd ed.). Sage.

Hyde, J. S. (2005). The gender similarities hypothesis. American Psychologist, 60(6), 581-592. https://doi.org/10.1037/0003-066X.60.6.581

Inglehart, R. F., \& Baker, W. E. (2000). Modernization, cultural change, and the persistence of traditional values. American Sociological Review, 65(1), 19-51. https://doi.org/10.2307/2657288

Inman, R. A., Silva, S. M. D., Bayoumi, R., \& Hanel, P. H. P. (2017). Cultural value orientations and alcohol consumption in 74 countries: A societal-level analysis. Frontiers in Psychology: Cultural Psychology, 8. https://doi.org/10.3389/fpsyg.2017.01963

Jen, M. H., Sund, E. R., Johnston, R., \& Jones, K. (2010). Trustful societies, trustful individuals, and health: An analysis of self-rated health and social trust using the World Value Survey. Health \& Place, 16(5), 1022-1029. https://doi.org/10.1016/j.healthplace.2010.06 .008

Lawrence, P. A. (2003). The politics of publication. Nature, 422(6929), 259-261. https://doi.org/10.1038/422259a

Leimu, R., \& Koricheva, J. (2005). What determines the citation frequency of ecological papers? Trends in Ecology \& Evolution, 20(1), 28-32. https://doi.org/10.1016/j.tree.2004.10.010

Lennon, J. C. (2019). Navigating academia as a PsyD student. Nature Human Behaviour. https://socialsciences.nature.com/channels/21 40-is-it-publish-or-perish/posts/52824-competi ng-in-the-world-of-academia-as-a-psyd-student

Lepori, B., Geuna, A., \& Mira, A. (2019). Scientific output scales with resources. A 
comparison of US and European universities. PLOS ONE, 14(10), e0223415. https://doi.org/10.1371/journal.pone.0223415

Levecque, K., Anseel, F., De Beuckelaer, A., Van der Heyden, J., \& Gisle, L. (2017). Work organization and mental health problems in $\mathrm{PhD}$ students. Research Policy, 46(4), 868-879. https://doi.org/10.1016/j.respol.2017.02.008

Leydesdorff, L., \& Milojević, S. (2013). Scientometrics. ArXiv:1208.4566 [Cs]. http://arxiv.org/abs/1208.4566

Lourenco, S. F., \& Tasimi, A. (2020). No Participant Left Behind: Conducting Science During COVID19. Trends in Cognitive Sciences, 24(8), 583-584. https://doi.org/10.1016/j.tics.2020.05.003

MacKinnon, D. P., Lockwood, C. M., Hoffman, J. M., West, S. G., \& Sheets, V. (2002). A comparison of methods to test mediation and other intervening variable effects. Psychological Methods, 7(1), 83.

May, K., \& Hittner, J. B. (1997). Tests for comparing dependent correlations revisited: A Monte Carlo study. The Journal of Experimental Education, 65, 257-269.

McKiernan, E. C., Bourne, P. E., Brown, C. T., Buck, S., Kenall, A., Lin, J., McDougall, D., Nosek, B. A., Ram, K., Soderberg, C. K., Spies, J. R., Thaney, K., Updegrove, A., Woo, K. H., \& Yarkoni, T. (2016). How open science helps researchers succeed. ELife, 5, e16800. https://doi.org/10.7554/eLife.16800

Michel, J.-B., Shen, Y. K., Aiden, A. P., Veres, A., Gray, M. K., Team, T. G. B., Pickett, J. P., Hoiberg, D., Clancy, D., Norvig, P., Orwant, J., Pinker, S., Nowak, M. A., \& Aiden, E. L. (2011). Quantitative analysis of culture using millions of digitized books. Science, 331(6014), 176-182. https://doi.org/10.1126/science.1199644

Moher, D., Liberati, A., Tetzlaff, J., \& Altman, D. G. (2009). Preferred reporting items for systematic reviews and meta-analyses: The PRISMA statement. Annals of Internal Medicine, 151(4), 264-269. https://doi.org/10.7326/0003-4819151-4-200908180-00135

Morris, T. P., White, I. R., \& Crowther, M. J. (2019). Using simulation studies to evaluate statistical methods. Statistics in Medicine, 38(11), 2074-2102. https://doi.org/10.1002/sim.8086

Nosek, B. A., Smyth, F. L., Sriram, N., Lindner, N. M., Devos, T., Ayala, A., Bar-Anan, Y., Bergh, R., Cai, H., Gonsalkorale, K., Kesebir, S., Maliszewski, N., Neto, F., Olli, E., Park, J., Schnabel, K., Shiomura, K., Tulbure, B. T., Wiers, R. W., ... Greenwald, A. G. (2009). National differences in gender-science stereotypes predict national sex differences in science and math achievement. Proceedings of the National Academy of Sciences, 106(26), 10593-10597. https://doi.org/10.1073/pnas.0809921106

Nuijten, M. B., Hartgerink, C. H. J., Assen, M. A. L. M. van, Epskamp, S., \& Wicherts, J. M. (2015). The prevalence of statistical reporting errors in psychology (1985-2013). Behavior Research Methods, 1-22. https://doi.org/10.3758/s13428-015-06642

Ondish, P., \& Stern, C. (2017). Liberals possess more national consensus on political attitudes in the United States: An examination across 40 years. Social Psychological and Personality Science, 9(8), 935-943. https://doi.org/10.1177/1948550617729410

Patsopoulos, N. A., Analatos, A. A., \& Ioannidis, J. P. A. (2005). Relative citation impact of various study designs in the health sciences. JAMA, 293(19), 2362-2366. https://doi.org/10.1001/jama.293.19.2362

Paxton, A., \& Griffiths, T. L. (2017). Finding the traces of behavioral and cognitive processes in big data and naturally occurring datasets. Behavior Research Methods, 49(5), 1630-1638. https://doi.org/10.3758/s13428-017-0874-x

Plavén-Sigray, P., Matheson, G. J., Schiffler, B. C., \& Thompson, W. H. (2017). Research: The readability of scientific texts is decreasing over time. ELife, 6, e27725. https://doi.org/10.7554/eLife.27725

Preis, T., Moat, H. S., \& Stanley, H. E. (2013). Quantifying Trading Behavior in Financial Markets Using Google Trends. Scientific Reports, 3. https://doi.org/10.1038/srep01684

Quintana, D. S. (2015). From pre-registration to publication: A non-technical primer for conducting a meta-analysis to synthesize correlational data. Frontiers in Psychology, 6. https://doi.org/10.3389/fpsyg.2015.01549

Reinhart, M. (2009). Peer review of grant applications in biology and medicine. Reliability, fairness, and validity. Scientometrics, 81(3), 789-809. https://doi.org/10.1007/s11192-008-2220-7

Rosinger, A. Y., \& Ice, G. (2019). Secondary data analysis to answer questions in human biology. American Journal of Human Biology, 31(3), e23232. https://doi.org/10.1002/ajhb.23232

Rudnev, M., \& Vauclair, C.-M. (2018). The link between personal values and frequency of drinking depends on cultural values: A cross-level interaction approach. Frontiers in Psychology, 9. https://doi.org/10.3389/fpsyg.2018.01379

Schmidt-Catran, A. W., \& Fairbrother, M. (2016). 
The random effects in multilevel models: Getting them wrong and getting them right. European Sociological Review, 32(1), 23-38. https://doi.org/10.1093/esr/jcv090

Schwartz, S. H. (2006). A theory of cultural value orientations: Explication and applications. Comparative Sociology, 5(2), 137-182. https://doi.org/10.1163/1569133067786673 57

Sherif, V. (2018). Evaluating preexisting qualitative research data for secondary analysis. Forum Qualitative Sozialforschung / Forum: Qualitative Social Research, 19(2). https://doi.org/10.17169/fqs19.2.2821

Simonsohn, U., Simmons, J. P., \& Nelson, L. D. (2015). Better P-curves: Making Pcurve analysis more robust to errors, fraud, and ambitious P-hacking, a Reply to Ulrich and Miller (2015). Journal of Experimental Psychology: General, 144(6), 1146-1152. https://doi.org/10.1037/xge0000104

Smaldino, P. E. (2020). How to translate a verbal theory into a formal model. https://files.osf.io/v1/resources/n7qsh/provid ers/osfstorage/5ecd62d2aeeb6d01d6087b01? format $=$ pdf\&action $=$ download\&direct $\&$ versi on $=2$

Smith, L. G. E., McGarty, C., \& Thomas, E. F. (2018). After Aylan Kurdi: How tweeting about death, threat, and harm predict increased expressions of solidarity with refugees over time. Psychological Science, 29(4), 623-634. https://doi.org/10.1177/0956797617741107

Sparks, S. (2019). How to find international collaborators for your research. British Council. https://www.britishcouncil.org/voicesmagazine/how-to-find-internationalcollaborators-for-your-research

Stanley, T. D., \& Doucouliagos, H. (2014). Metaregression approximations to reduce publication selection bias. Research Synthesis Methods, 5(1), 60-78. https://doi.org/10.1002/jrsm.1095

Stewart, L., Moher, D., \& Shekelle, P. (2012). Why prospective registration of systematic reviews makes sense. Systematic Reviews, 1(1), 7. https://doi.org/10.1186/2046-4053-1-7

Teixeira da Silva, J. A., \& Memon, A. R. (2017). CiteScore: A cite for sore eyes, or a valuable, transparent metric? Scientometrics, 111(1), 553-556. https://doi.org/10.1007/s11192-017-2250-0

United Nations Developmental Programme. (2014). Human Developmental Report: Human Development Index (HDI). http://hdr.undp.org/en/data

Urbanska, K. (2019). Oh no, I haven't published:
Navigating the job market without a publication record. Nature Human Behaviour. https://socialsciences.nature.com/users/30163 3-karolina-urbanska/posts/54645-oh-no-i-hav ent-published-navigating-the-job-market-wi thout-apublication-record

Van den Akker, O., Weston, S. J., Campbell, L., Chopik, W. J., Damian, R. I., Davis-Kean, P., Hall, A. N., Kosie, J. E., Kruse, E. T., Olsen, J., Ritchie, S. J., Valentine, K. D., van 't Veer, A. E., \& Bakker, M. (2019). Preregistration of secondary data analysis: A template and tutorial [Preprint]. PsyArXiv. https://doi.org/10.31234/osf.io/hvfmr

Van der Veer, T., Baars, J. E., Birnie, E., \& Hamberg, P. (2015). Citation analysis of the 'Big Six' journals in Internal Medicine. European Journal of Internal Medicine, 26(6), 458-459. https://doi.org/10.1016/j.ejim.2015.05.017

Van Lange, P. A. M. (2013). What we should expect from theories in social psychology: Truth, abstraction, progress, and applicability as standards (TAPAS). Personality and Social Psychology Review, 17(1), 40-55. https://doi.org/10.1177/1088868312453088

Viechtbauer, W. (2010). Conducting meta-analyses in $\mathrm{R}$ with the metafor package. Journal of Statistical Software, 36(3), 1-48.

Waagmeester, A., Stupp, G., Burgstaller-Muehlbacher, S., Good, B. M., Griffith, M., Griffith, O. L., Hanspers, K., Hermjakob, H., Hudson, T. S., Hybiske, K., Keating, S. M., Manske, M., Mayers, M., Mietchen, D., Mitraka, E., Pico, A. R., Putman, T., Riutta, A., Queralt-Rosinach, N., ... $\mathrm{Su}$, A. I. (2020). Wikidata as a knowledge graph for the life sciences. ELife, 9, e52614. https://doi.org/10.7554/eLife.52614

Wang, Y., \& Bowers, A. J. (2016). Mapping the field of educational administration research: A journal citation network analysis. Journal of Educational Administration, 54(3). https://doi.org/10.1108/JEA02-2015-0013

Weaver, B. P., \& Hamada, M. S. (2016). Quality quandaries: A gentle introduction to Bayesian statistics. Quality Engineering, 28(4), 508-514. https://doi.org/10.1080/08982112.2016.1167 220

Webb, T. L., Miles, E., \& Sheeran, P. (2012). Dealing with feeling: A meta-analysis of the effectiveness of strategies derived from the process model of emotion regulation. Psychological Bulletin, 138(4), 775-808. https://doi.org/10.1037/a0027600

Weissgerber, T. L., Garovic, V. D., Savic, M., Winham, S. J., \& Milic, N. M. (2016). From Static to Interac- 
tive: Transforming Data Visualization to Improve Transparency. PLOS Biology, 14(6), e1002484. https://doi.org/10.1371/journal.pbio.1002484

Weissgerber, T. L., Milic, N. M., Winham, S. J.,
\& Garovic, V. D. (2015). Beyond bar and line graphs: Time for a new data presentation paradigm. PLoS Biology, 13(4), e1002128. https://doi.org/10.1371/journal.pbio.1002128 